\title{
Pricing Options with Credit Risk in Markovian Regime-Switching Markets
}

\author{
Jinzhi $\mathrm{Li}^{1}$ and Shixia $\mathrm{Ma}^{2}$ \\ ${ }^{1}$ College of Sciences, Minzu University of China, Beijing 100081, China \\ ${ }^{2}$ College of Sciences, Hebei University of Technology, Tianjin 300401, China \\ Correspondence should be addressed to Jinzhi Li; lijinzhil18@gmail.com
}

Received 16 February 2013; Revised 16 May 2013; Accepted 20 May 2013

Academic Editor: Shan Zhao

Copyright (c) $2013 \mathrm{~J}$. Li and S. Ma. This is an open access article distributed under the Creative Commons Attribution License, which permits unrestricted use, distribution, and reproduction in any medium, provided the original work is properly cited.

\begin{abstract}
This paper investigates the valuation of European option with credit risk in a reduced form model when the stock price is driven by the so-called Markov-modulated jump-diffusion process, in which the arrival rate of rare events and the volatility rate of stock are controlled by a continuous-time Markov chain. We also assume that the interest rate and the default intensity follow the Vasicek models whose parameters are governed by the same Markov chain. We study the pricing of European option and present numerical illustrations.
\end{abstract}

\section{Introduction}

Pricing options with credit risk is an important topic in finance from both theoretical and practical perspectives. Credit risk refers to an investor's risk that a borrower will default on making payments as promised. There are basically two kinds of models to describe the default: structural models and reduced form models. The structural approach was firstly introduced by Merton [1] who investigated European option pricing for modeling single corporate default. The approach is further extended by recent literature: see Ammann [2] and Klein and Inglis [3]. Another tractable approach is called reduced form model, which models the intensity of arrival of default events directly. The reduced form models can be seen in Artzner and Freddy [4], Duffie and Singleton [5], Duffie and Gârleanu [6], and Leung and Kwok [7] and are developed extensively by Su and Wang [8].

Recently, Markovian regime-switching models have attracted attention among researchers and practitioners in economics and mathematics. Elliott et al. [9] introduce a self-calibrating model for short-term interest rate by assuming that the short rate is governed by a finite state space Markov process. Elliott et al. [10] and Elliott and Osakwe [11] use Markov-modulated market parameters to capture the time inhomogeneity generated by the financial market.
Elliott et al. [12] perform the valuation of option under a generalized Markov-modulated jump-diffusion model. Siu et al. [13] consider the pricing currency options under two-factor Markov-modulated stochastic volatility models. Bo et al. [14] derive the valuation of currency option when the spot foreign exchange rates follow Markov-modulated jump-diffusion model.

In this paper, we investigate the valuation of European option with credit risk in a reduced form model in Markovian regime-switching markets. We assume that the recovery rate is constant; that is, when the writer of the option defaults, a specified constant fraction times the payoff will be paid at maturity. In order to incorporate both rare events and time-inhomogeneity in finance market, we model the stock price by the so-called Markov-modulated jump-diffusion process, in which rare events are described as a compound Poisson process and the arrival rate of Poisson process and the volatility rate of stock are governed by a continuous-time Markov chain. The states of Markov chain can be interpreted as the states of the market. The transitions of the states of the market may describe changes of economy, finance, business cycles, and other conditions. In addition, we assume that the interest rate and the default intensity both follow the Vasicek models and the parameters of models are correlated with the same Markov chain. By the method of changing measures, 
we obtain the closed form formula for the valuation of the European option.

The rest of this paper is organized as follows. Section 2 presents the model description. In Section 3, by applying Girsanov's measure changing theorem, we derive the formula of the pricing of European-style call option. We provide numerical analysis in Section 4. The concluding remarks are contained in Section 5.

\section{The Model Description}

Let $(\Omega, \mathscr{F}, P)$ be a complete probability space, where $P$ is a neutral-risk probability measure. Define $\xi=\left\{\xi_{t}, t \geq 0\right\}$ on $(\Omega, \mathscr{F}, P)$ as a continuous-time, finite state Markov chain with $n$-state space $E$. We interpret the state of $\xi$ as the states of the economy as follows (Elliott et al. [10] and Elliott and Osakwe [11]). Without loss of generality, we take the state space of $\xi$ to be a finite set of unite vectors $\left\{e_{1}, e_{2}, \ldots, e_{n}\right\}$ with $e_{i}=(0, \ldots, 1, \ldots, 0) \in R^{n}$. And $\xi$ has the following semimartingale representation:

$$
d \xi_{t}=Q \xi_{t} d t+d M_{t}
$$

where $Q=\left(q_{i j}\right)_{i, j=1,2, \ldots, n}$ is $Q$-matrix of $\xi$ and $M=\left\{M_{t}\right\}_{0 \leq t \leq T}$ is an $R^{n}$-valued martingale with respect to the filtration generated by $\left\{\xi_{t}, 0 \leq t \leq T\right\}$ under $P$. Suppose that the stock price $S_{t}$ and interest rate $r_{t}$ satisfy the following stochastic differential equations (SDE):

$$
\begin{gathered}
\frac{d S_{t}}{S_{t-}}=\left(r_{t}-k v_{t}\right) d t+\sigma_{1 t} d W_{1 t}+\left(e^{Y_{t-}}-1\right) d N(t), \\
d r_{t}=\left(a_{t}-b_{t} r_{t}\right) d t+\sigma_{2 t} d W_{2 t},
\end{gathered}
$$

where $W_{1 t}, W_{2 t}$ are standard Brownian motions and $\sigma_{1 t}, \sigma_{2 t}$ are the stochastic volatility of the stock and the interest rate, respectively. $\left\{N_{t}\right\}_{0 \leq t \leq T}$ is Poisson process with the stochastic jump intensity $\left\{v_{t}\right\}_{0 \leq t \leq T}$, and the jump amplitude is controlled by $\left\{Y_{t}\right\} . Y_{s}$ and $Y_{t}$ for $s \neq t$ independently identify distribution, and write $k=E e^{Y_{i}}-1$. Moreover, $Y_{t}, N_{t}$ are assumed to be mutually independent. $\sigma_{1 t}, \sigma_{2 t}, v_{t}, a_{t}, b_{t}$ are controlled by $\xi_{t}$, that is,

$$
\begin{array}{cl}
\sigma_{1 t}=\left\langle\sigma_{1}, \xi_{t}\right\rangle, & \sigma_{1}=\left(\sigma_{11}, \sigma_{12}, \ldots, \sigma_{1 n}\right) \in(0, \infty)^{n}, \\
\sigma_{2 t}=\left\langle\sigma_{2}, \xi_{t}\right\rangle, & \sigma_{2}=\left(\sigma_{21}, \sigma_{22}, \ldots, \sigma_{2 n}\right) \in(0, \infty)^{n}, \\
v_{t}=\left\langle\nu, \xi_{t}\right\rangle, & v=\left(v_{1}, v_{2}, \ldots, v_{n}\right) \in(0, \infty)^{n}, \\
a_{t}=\left\langle a, \xi_{t}\right\rangle, & a=\left(a_{1}, a_{2}, \ldots, a_{n}\right) \in(0, \infty)^{n}, \\
b_{t}=\left\langle b, \xi_{t}\right\rangle, & b=\left(b_{1}, b_{2}, \ldots, b_{n}\right) \in(0, \infty)^{n},
\end{array}
$$

where $\langle\cdot, \cdot\rangle$ denotes the inner product in $R^{n}$. Let $\tau$ denote the default time of the writer of the option with default intensity process $\lambda_{t}$, and $\lambda_{t}$ is given by

$$
d \lambda_{t}=\left(\alpha_{t}-\beta_{t} \lambda_{t}\right) d t+\sigma_{3 t} d W_{3 t},
$$

where $W_{3 t}$ is standard Brownian motion and $\sigma_{3 t}$ is the stochastic volatility of default intensity. $\sigma_{3 t}, \alpha_{t}$, and $\beta_{t}$ are also controlled by $\xi_{t}$ and satisfy

$$
\begin{gathered}
\sigma_{3 t}=\left\langle\sigma_{3}, \xi_{t}\right\rangle, \quad \sigma_{3}=\left(\sigma_{31}, \sigma_{32}, \ldots, \sigma_{3 n}\right) \in(0, \infty)^{n}, \\
\alpha_{t}=\left\langle\alpha, \xi_{t}\right\rangle, \quad \alpha=\left(\alpha_{1}, \alpha_{2}, \ldots, \alpha_{n}\right) \in(0, \infty)^{n}, \\
\beta_{t}=\left\langle\beta, \xi_{t}\right\rangle, \quad \beta=\left(\beta_{1}, \beta_{2}, \ldots, \beta_{n}\right) \in(0, \infty)^{n} .
\end{gathered}
$$

Moreover, we assume that $N_{t}, Y_{t}$ are independent of $W_{1 t}, W_{2 t}$, and $W_{3 t}$ and the covariance matrix of the Brownian motion $\left(W_{1 t}, W_{2 t}, W_{3 t}\right)$ is

$$
\left(\begin{array}{ccc}
1 & \rho_{12} & \rho_{13} \\
\rho_{12} & 1 & \rho_{23} \\
\rho_{13} & \rho_{23} & 1
\end{array}\right) t
$$

The filtration $\mathscr{F}_{t}$ is generated by $\mathscr{F}_{t}=\mathscr{F}_{t}^{S} \vee \mathscr{F}_{t}^{r} \vee \mathscr{F}_{t}^{\lambda} \vee \mathscr{F}_{T}^{\xi} \vee$ $\mathscr{H}_{t}$, where $\mathscr{F}_{t}^{S}=\sigma\left(S_{s}, 0 \leq s \leq t\right), \mathscr{F}_{t}^{r}=\sigma\left(r_{s}, 0 \leq s \leq t\right)$, $\mathscr{F}_{t}^{\lambda}=\sigma\left(\lambda_{s}, 0 \leq s \leq t\right), \mathscr{F}_{t}^{\xi}=\sigma\left(\xi_{s}, 0 \leq s \leq t\right)$, and $\mathscr{H}_{t}=$ $\sigma\left(1_{(\tau \leq s)}, s \leq t\right)$.

\section{Pricing Options with Credit Risk}

We consider the case of a European call option with credit risk. Assume that the recovery rate is a constant $w$. When the seller of option defaults, the payoff is given by $w$ times the payoff of the default-free option at maturity. Therefore, by the risk neutral pricing theorem, the valuation of the European call option at time $t$, with strike price $K$ and maturity $T$, is given by

$$
\begin{aligned}
C & (t, T) \\
& =E\left[e^{-\int_{t}^{T} r_{s} d s}\left(w\left(S_{T}-K\right)^{+} 1_{(\tau \leq T)}+\left(S_{T}-K\right)^{+} 1_{(\tau>T)}\right) \mid \mathscr{F}_{t}\right] \\
& =E\left[e^{-\int_{t}^{T} r_{s} d s}\left(w\left(S_{T}-K\right)^{+}+(1-w)\left(S_{T}-K\right)^{+} 1_{(\tau>T)}\right) \mid \mathscr{F}_{t}\right] .
\end{aligned}
$$

Following Lando [15],

$$
\begin{aligned}
& E\left[e^{-\int_{t}^{T} r_{s} d s}\left(S_{T}-K\right)^{+} 1_{(\tau>T)} \mid \mathscr{F}_{t}\right] \\
& \quad=1_{(\tau>t)} E\left[e^{-\int_{t}^{T}\left(r_{s}+\lambda_{s}\right) d s}\left(S_{T}-K\right)^{+} \mid \mathscr{F}_{t}\right] .
\end{aligned}
$$

We can obtain the following expression:

$$
\begin{aligned}
C(t, T)= & w E\left[e^{-\int_{t}^{T} r_{s} d s}\left(S_{T}-K\right)^{+} \mid \mathscr{F}_{t}\right] \\
& +(1-w) 1_{(\tau>t)} E\left[e^{-\int_{t}^{T}\left(r_{s}+\lambda_{s}\right) d s}\left(S_{T}-K\right)^{+} \mid \mathscr{F}_{t}\right] \\
= & I_{1}+I_{2} .
\end{aligned}
$$

Next, we calculate $I_{1}$ and $I_{2}$, respectively. For $s>t$, we have

$$
r_{s}=e^{-\int_{t}^{s} b_{u} d u} r_{t}+\int_{t}^{s} e^{-\int_{v}^{s} b_{u} d u} a_{v} d v+\int_{t}^{s} e^{-\int_{v}^{s} b_{u} d u} \sigma_{2 v} d W_{2 v} .
$$


Integrated from $t$ to $T$ in both sides of (10),

$$
\begin{aligned}
\int_{t}^{T} r_{s} d s= & \int_{t}^{T} e^{-\int_{t}^{s} b_{u} d u} d s r_{t}+\int_{t}^{T} a_{v} d v \int_{v}^{T} e^{-\int_{v}^{s} b_{u} d u} d s \\
& +\int_{t}^{T} \sigma_{2 v} d W_{2 v} \int_{v}^{T} e^{-\int_{v}^{s} b_{u} d u} d s .
\end{aligned}
$$

Let $M(x, y, T)=\int_{y}^{T} e^{-\int_{y}^{s} x_{u} d u} d s$; then

$$
\begin{aligned}
\int_{t}^{T} r_{s} d s= & M(b, t, T) r_{t}+\int_{t}^{T} a_{v} M(b, v, T) d v \\
& +\int_{t}^{T} \sigma_{2 v} M(b, v, T) d W_{2 v} .
\end{aligned}
$$

Similarly,

$$
\begin{aligned}
\int_{t}^{T} \lambda_{s} d s= & M(\beta, t, T) \lambda_{t}+\int_{t}^{T} \alpha_{v} M(\beta, v, T) d v \\
& +\int_{t}^{T} \sigma_{3 v} M(\beta, v, T) d W_{3 v} .
\end{aligned}
$$

From (12) and (13), we have that

$$
\begin{aligned}
Z(t, T):=E\left[\exp \left\{-\int_{t}^{T}\left(r_{s}+\lambda_{s}\right) d s\right\} \mid \mathscr{F}_{t}\right] \\
=\exp \left\{-M(b, t, T) r_{t}-M(\beta, t, T) \lambda_{t}\right. \\
\quad-\int_{t}^{T} a_{u} M(b, u, T) d u \\
\left.\quad-\int_{t}^{T} \alpha_{u} M(\beta, u, T) d u\right\} \\
\times \exp \left\{\frac{1}{2} \int_{t}^{T} \sigma_{2 u}^{2} M^{2}(b, u, T) d u\right. \\
\left.\quad+\frac{1}{2} \int_{t}^{T} \sigma_{3 u}^{2} M^{2}(\beta, u, T) d u\right\} \\
\times \exp \left\{\rho_{23} \int_{t}^{T} \sigma_{2 u} \sigma_{3 u} M(b, u, T) M(\beta, u, T) d u\right\} .
\end{aligned}
$$

Thus, we can define the probability measure $Q$ by

$$
\begin{aligned}
\left.\frac{d Q}{d P}\right|_{\mathscr{F}_{t}}= & \frac{\exp \left\{-\int_{t}^{T}\left(r_{s}+\lambda_{s}\right) d s\right\}}{E\left[\exp \left\{-\int_{t}^{T}\left(r_{s}+\lambda_{s}\right) d s\right\} \mid \mathscr{F}_{t}\right]} \\
= & \exp \left\{-\frac{1}{2} \int_{t}^{T} \sigma_{2 u}^{2} M^{2}(b, u, T) d u\right. \\
& \left.-\frac{1}{2} \int_{t}^{T} \sigma_{3 u}^{2} M^{2}(\beta, u, T) d u\right\} \\
& \times \exp \left\{-\rho_{23} \int_{t}^{T} \sigma_{2 u} \sigma_{3 u} M(b, u, T) M(\beta, u, T) d u\right\} \\
\times & \quad \exp \left\{-\int_{t}^{T} \sigma_{2 v} M(b, v, T) d W_{2 v}\right. \\
& \left.\quad-\int_{t}^{T} \sigma_{3 v} M(\beta, v, T) d W_{3 v}\right\} .
\end{aligned}
$$

By Girsanov's theorem,

$$
\begin{aligned}
& d W_{2 t}^{\mathrm{Q}}=d W_{2 t}+\sigma_{2 t} M(b, t, T) d t+\rho_{23} \sigma_{3 t} M(\beta, t, T) d t, \\
& d W_{3 t}^{\mathrm{Q}}=d W_{3 t}+\sigma_{3 t} M(\beta, t, T) d t+\rho_{23} \sigma_{2 t} M(b, t, T) d t, \\
& d W_{1 t}^{\mathrm{Q}}=d W_{1 t}+\rho_{12} M_{1}(t) d t+\rho_{13} M_{2}(t) d t,
\end{aligned}
$$

where

$$
\begin{aligned}
& M_{1}(t)=\sigma_{2 t} M(b, t, T)+\rho_{23} \sigma_{3 t} M(\beta, t, T), \\
& M_{2}(t)=\sigma_{3 t} M(\beta, t, T)+\rho_{23} \sigma_{2 t} M(b, t, T) .
\end{aligned}
$$

$W_{1 t}^{\mathrm{Q}}, W_{2 t}^{\mathrm{Q}}$, and $W_{3 t}^{\mathrm{Q}}$ are standard Brownian motions under probability measure $\mathrm{Q}$, and $\left(W_{1 t}^{\mathrm{Q}}, W_{2 t}^{\mathrm{Q}}, W_{3 t}^{\mathrm{Q}}\right)$ has the same covariance matrix as $\left(W_{1 t}, W_{2 t}, W_{3 t}\right)$. Therefore,

$$
\begin{aligned}
& E\left[e^{-\int_{t}^{T}\left(r_{s}+\lambda_{s}\right) d s}\left(S_{T}-K\right)^{+} \mid \mathscr{F}_{t}\right] \\
& =Z(t, T) E^{Q}\left(\left(S_{T}-K\right)^{+} \mid \mathscr{F}_{t}\right) .
\end{aligned}
$$

In addition,

$$
\begin{aligned}
E^{\mathrm{Q}} & \left(\left(S_{T}-K\right)^{+} \mid \mathscr{F}_{t}\right) \\
& =E^{\mathrm{Q}}\left(S_{T} 1_{\left(S_{T} \geq K\right)} \mid \mathscr{F}_{t}\right)-K E^{\mathrm{Q}}\left(1_{\left(S_{T} \geq K\right)} \mid \mathscr{F}_{t}\right) .
\end{aligned}
$$

By the solution of SDE (2),

$$
\begin{aligned}
S_{T}=S_{t} \exp \left\{\int_{t}^{T}\left(r_{s}-k v_{s}-\frac{1}{2} \sigma_{1 s}^{2}\right) d s\right. \\
\left.+\int_{t}^{T} \sigma_{1 s} d W_{1 s}+\int_{t}^{T} Y_{s-} d N_{s}\right\} .
\end{aligned}
$$


Under $Q$,

$$
\begin{aligned}
S_{T}=S_{t} \exp \left\{\Lambda(t, T)+\int_{t}^{T} \sigma_{1 s} d W_{1 s}^{\mathrm{Q}}\right. & \\
& \left.+\int_{t}^{T} \sigma_{2 s} M(b, s, T) d W_{2 s}^{\mathrm{Q}}+\int_{t}^{T} Y_{s-} d N_{s}\right\},
\end{aligned}
$$

where

$$
\begin{aligned}
\Lambda(t, T)= & -\int_{t}^{T}\left(k v_{s}+\frac{1}{2} \sigma_{1 s}^{2}\right) d s+M(b, t, T) r_{t} \\
& +\int_{t}^{T} a_{s} M(b, s, T) d s-\int_{t}^{T} \sigma_{2 s}^{2} M^{2}(b, s, T) d s \\
& -\rho_{12} \int_{t}^{T} \sigma_{1 s} M_{1}(s) d s-\rho_{13} \int_{t}^{T} \sigma_{1 s} M_{2}(s) d s \\
& -\rho_{23} \int_{t}^{T} \sigma_{2 s} \sigma_{3 s} M(b, s, T) M(\beta, s, T) d s .
\end{aligned}
$$

So,

$$
\begin{array}{r}
P^{\mathrm{Q}}\left(S_{T} \geq K \mid \mathscr{F}_{t}\right) \\
=P^{\mathrm{Q}}\left(\int_{t}^{T} \sigma_{1 s} d W_{1 s}^{\mathrm{Q}}+\int_{t}^{T} \sigma_{2 s} M(b, s, T) d W_{2 s}^{\mathrm{Q}}\right. \\
\left.\geq \ln \left(K / S_{t}\right)-\Lambda(t, T)-\int_{t}^{T} Y_{s} d N_{s}\right) \\
=P^{\mathrm{Q}}\left(\frac{\int_{t}^{T} \sigma_{1 s} d W_{1 s}^{\mathrm{Q}}+\int_{t}^{T} \sigma_{2 s} M(b, s, T) d W_{2 s}^{\mathrm{Q}}}{\sqrt{\Delta}}\right. \\
\left.\leq \frac{\ln \left(S_{t} / K\right)+\Lambda(t, T)+\int_{t}^{T} Y_{s} d N_{s}}{\sqrt{\Delta}}\right) \\
=\sum_{n=0}^{\infty} \frac{\left(\int_{t}^{T} v_{s} d s\right)^{n}}{n !} e^{-\int_{t}^{T} v_{s} d s} E\left(N\left(d_{1}\right)\right),
\end{array}
$$

where

$$
\begin{gathered}
\Delta=\int_{t}^{T} \sigma_{1 s}^{2} d s+\int_{t}^{T} \sigma_{2 s}^{2} M^{2}(b, s, T) d s \\
+2 \rho_{12} \int_{t}^{T} \sigma_{1 s} \sigma_{2 s} M(b, s, T) d s, \\
d_{1}=\frac{\ln \left(S_{t} / K\right)+\Lambda(t, T)+\sum_{j=1}^{n} Y_{j}}{\sqrt{\Delta}} .
\end{gathered}
$$

$E(\cdot)$ is the expectation of $Y_{j}$ under $P$, and $N(\cdot)$ is distribution function of standard normal distribution. On the other hand, let

$$
\begin{aligned}
\mathscr{L}(t, T) & :=E^{\mathrm{Q}}\left(S_{T} \mid \mathscr{F}_{t}\right) \\
= & S_{t} e^{\Lambda(t, T)} \exp \left\{\frac{1}{2} \int_{t}^{T} \sigma_{1 s}^{2} d s+\frac{1}{2} \int_{t}^{T} \sigma_{2 s}^{2} M^{2}(b, s, T) d s\right\} \\
& \times \exp \left\{\rho_{12} \int_{t}^{T} \sigma_{1 s} \sigma_{2 s} M(b, s, T) d s+k \int_{t}^{T} v_{s} d s\right\} .
\end{aligned}
$$

We can define the probability measure $R$ as follows:

$$
\begin{aligned}
\left.\frac{d R}{d Q}\right|_{\mathscr{F}_{t}}= & \frac{S_{T}}{E^{\mathrm{Q}}\left(S_{T} \mid \mathscr{F}_{t}\right)} \\
= & \exp \left\{\int_{t}^{T} \sigma_{1 s} d W_{1 s}^{\mathrm{Q}}+\int_{t}^{T} \sigma_{2 s} M(b, s, T) d W_{2 s}^{\mathrm{Q}}\right. \\
& \left.\quad-\frac{1}{2} \int_{t}^{T} \sigma_{1 s}^{2} d s\right\} \\
& \times \exp \left\{-\frac{1}{2} \int_{t}^{T} \sigma_{2 s}^{2} M^{2}(b, s, T) d s\right. \\
& \left.\quad-\rho_{12} \int_{t}^{T} \sigma_{1 s} \sigma_{2 s} M(b, s, T) d s\right\} \\
& \times \exp \left\{\int_{t}^{T} Y_{s} d N_{s}-k \int_{t}^{T} v_{s} d s\right\} .
\end{aligned}
$$

By Girsanov's theorem, we have that

$$
\begin{aligned}
& d W_{1 t}^{R}=d W_{1 t}^{\mathrm{Q}}-\sigma_{1 t} d t-\rho_{12} \sigma_{2 t} M(b, t, T) d t, \\
& d W_{2 t}^{R}=d W_{2 t}^{\mathrm{Q}}-\sigma_{2 t} M(b, t, T) d t-\rho_{12} \sigma_{1 t} d t,
\end{aligned}
$$

where, under $R, W_{1 t}^{R}, W_{2 t}^{R}$ are standard Brownian motions and their correlation coefficient is $\rho_{12}, N_{t}$ is Poisson process with intensity $(k+1) v_{t}$, and the density function of $Y_{1}$ is $e^{y} f(y) /(k+1)$, where $f(\cdot)$ is the density function of $Y_{1}$ under $P$. Since, under $R$,

$$
\begin{aligned}
S_{T}=S_{t} \exp \{ & \Lambda(t, T)+\Delta+\int_{t}^{T} \sigma_{1 s} d W_{1 s}^{R} \\
& \left.+\int_{t}^{T} \sigma_{2 s} M(b, s, T) d W_{2 s}^{R}+\int_{t}^{T} Y_{s-} d N_{s}\right\},
\end{aligned}
$$


then

$$
\begin{aligned}
E^{Q} & \left(S_{T} 1_{\left(S_{T} \geq K\right)} \mid \mathscr{F}_{t}\right) \\
= & \mathscr{L}(t, T) P^{R}\left(S_{T} \geq K \mid \mathscr{F}_{t}\right) \\
= & \mathscr{L}(t, T) P^{R}\left(\int_{t}^{T} \sigma_{1 s} d W_{1 s}^{R}+\int_{t}^{T} \sigma_{2 s} M(b, s, T) d W_{2 s}^{R}\right. \\
& \left.\geq \ln \left(\frac{K}{S_{t}}\right)-\Lambda(t, T)-\Delta-\int_{t}^{T} Y_{s} d N_{s}\right) \\
= & \mathscr{L}(t, T) P^{R}\left(\frac{\int_{t}^{T} \sigma_{1 s} d W_{1 s}^{R}+\int_{t}^{T} \sigma_{2 s} M(b, s, T) d W_{2 s}^{R}}{\sqrt{\Delta}}\right. \\
& \leq \frac{\ln \left(S_{t} / K\right)+\Lambda(t, T)+\Delta+\int_{t}^{T} Y_{s} d N_{s}}{\sqrt{\Delta}} \\
= & \mathscr{L}(t, T) \sum_{n=0}^{\infty} \frac{\left(\int_{t}^{T} v_{s} d s\right)^{n}}{n !} e^{-\int_{t}^{T} v_{s} d s} E^{R}\left(N\left(d_{2}\right)\right),
\end{aligned}
$$

where

$$
\begin{aligned}
d_{2} & =\frac{\ln \left(S_{t} / K\right)+\Lambda(t, T)+\Delta+\sum_{j=1}^{n} Y_{j}}{\sqrt{\Delta}} \\
& =d_{1}+\sqrt{\Delta}
\end{aligned}
$$

and $E^{R}(\cdot)$ is the expectation of $Y_{j}$ under $R$. By (23) and (29), we have the following lemma.

Lemma 1. Consider the Following:

$$
\begin{aligned}
& E\left[e^{-\int_{t}^{T}\left(r_{s}+\lambda_{s}\right) d s}\left(S_{T}-K\right)^{+} \mid \mathscr{F}_{t}\right] \\
& =Z(t, T) \\
& \quad \times \sum_{n=0}^{\infty} \frac{\left(\int_{t}^{T} v_{s} d s\right)^{n}}{n !} \\
& \quad \times e^{-\int_{t}^{T} v_{s} d s}\left[\mathscr{L}(t, T) E^{R}\left(N\left(d_{2}\right)\right)-K E\left(N\left(d_{1}\right)\right)\right] .
\end{aligned}
$$

In the following; in order to calculate $I_{1}$, we write

$$
\begin{aligned}
& E\left[e^{-\int_{t}^{T} r_{s} d s}\left(S_{T}-K\right)^{+} \mid \mathscr{F}_{t}\right] \\
&=E\left[e^{-\int_{t}^{T} r_{s} d s} S_{T} 1_{\left(S_{T} \geq K\right)} \mid \mathscr{F}_{t}\right] \\
&-K E\left[e^{-\int_{t}^{T} r_{s} d s} 1_{\left(S_{T} \geq K\right)} \mid \mathscr{F}_{t}\right] \\
&=I_{3}-I_{4} .
\end{aligned}
$$

Denote by $P(t, T)$ the value at time $t$ of a $T$ maturity zero coupon bond whose face value is 1 . Then

$$
P(t, T)=E\left[e^{-\int_{t}^{T} r_{s} d s} \mid \mathscr{F}_{t}\right]
$$

From (12),

$$
\begin{gathered}
P(t, T)=\exp \left\{-M(b, t, T) r_{t}-\int_{t}^{T} a_{v} M(b, v, T) d v\right. \\
\left.+\frac{1}{2} \int_{t}^{T} \sigma_{2 v}^{2} M^{2}(b, v, T) d v\right\}, \\
\frac{d P(t, T)}{P(t, T)}=r_{t} d t-M(b, t, T) \sigma_{2 t} d W_{2 t} .
\end{gathered}
$$

Define the Radon-Nikodym derivative given by

$$
\begin{aligned}
\frac{d Q^{T}}{d P} & =\frac{e^{-\int_{t}^{T} r_{s} d s}}{E\left[e^{-\int_{t}^{T} r_{s} d s} \mid \mathscr{F}_{t}\right]} \\
= & \exp \left\{-\int_{t}^{T} M(b, v, T) \sigma_{2 v} d W_{2 v}\right. \\
& \left.-\frac{1}{2} \int_{t}^{T} \sigma_{2 v}^{2} M^{2}(b, v, T) d v\right\},
\end{aligned}
$$

and by Girsanov's theorem, under $Q^{T}$,

$$
\begin{gathered}
d W_{2 t}^{Q^{T}}=d W_{2 t}+\sigma_{2 t} M(b, t, T) d t \\
d W_{1 t}^{Q^{T}}=d W_{1 t}+\rho_{12} \sigma_{2 t} M(b, t, T) d t .
\end{gathered}
$$

Thus $S_{T}$ can be rewritten as

$$
\begin{array}{r}
S_{T}=S_{t} \exp \left\{\mathscr{A}(t, T)+\int_{t}^{T} M(b, v, T) \sigma_{2 v} d W_{2 v}^{\mathrm{Q}^{T}}\right. \\
\left.+\int_{t}^{T} \sigma_{1 v} d W_{1 v}^{\mathrm{Q}^{T}}+\int_{t}^{T} Y_{s^{-}} d N_{s}\right\}
\end{array}
$$

where

$$
\begin{aligned}
\mathscr{A}(t, T)= & -\int_{t}^{T}\left(k v_{s}+\frac{1}{2} \sigma_{1 s}^{2}\right) d s+M(b, t, T) r_{t} \\
& +\int_{t}^{T} a_{s} M(b, s, T) d s-\int_{t}^{T} \sigma_{2 s}^{2} M^{2}(b, s, T) d s \\
& -\rho_{12} \int_{t}^{T} \sigma_{1 s} \sigma_{2 s} M(b, s, T) d s .
\end{aligned}
$$


Then

$$
\begin{array}{r}
P^{Q^{T}}\left(S_{T} \geq K \mid \mathscr{F}_{t}\right) \\
=P^{Q^{T}}\left(\int_{t}^{T} \sigma_{1 s} d W_{1 s}^{Q^{T}}+\int_{t}^{T} \sigma_{2 s} M(b, s, T) d W_{2 s}^{Q^{T}}\right. \\
\left.\geq \ln \left(\frac{K}{S_{t}}\right)-\mathscr{A}(t, T)-\int_{t}^{T} Y_{s-} d N_{s}\right) \\
=P^{Q^{T}}\left(\frac{\int_{t}^{T} \sigma_{1 s} d W_{1 s}^{Q^{T}}+\int_{t}^{T} \sigma_{2 s} M(b, s, T) d W_{2 s}^{Q^{T}}}{\sqrt{\Delta}}\right. \\
\left.\quad \leq \frac{\ln \left(S_{t} / K\right)+\mathscr{A}(t, T)+\int_{t}^{T} Y_{s-} d N_{s}}{\sqrt{\Delta}}\right) \\
=\sum_{n=0}^{\infty} \frac{\left(\int_{t}^{T} v_{s} d s\right)^{n}}{n !} e^{-\int_{t}^{T} v_{s} d s} E\left(N\left(d_{3}\right)\right),
\end{array}
$$

where $E(\cdot)$ is the expectation of $Y_{j}$ under $P$,

$$
d_{3}=\frac{\ln \left(S_{t} / K\right)+\mathscr{A}(t, T)+\sum_{j=1}^{n} Y_{j}}{\sqrt{\Delta}} .
$$

Therefore,

$$
\begin{aligned}
I_{4} & =K P(t, T) E^{Q^{T}}\left(1_{\left(S_{T} \geq K\right)} \mid \mathscr{F}_{t}\right) \\
& =K P(t, T) \sum_{n=0}^{\infty} \frac{\left(\int_{t}^{T} v_{s} d s\right)^{n}}{n !} e^{-\int_{t}^{T} v_{s} d s} E\left(N\left(d_{3}\right)\right) .
\end{aligned}
$$

Finally, let

$$
\begin{aligned}
A(t, T):= & E\left(e^{-\int_{t}^{T} r_{s} d s} S_{T} \mid \mathscr{F}_{t}\right) \\
= & S_{t} \exp \left\{-\int_{t}^{T}\left(k v_{s}+\frac{1}{2} \sigma_{1 s}^{2}\right) d s\right. \\
& \left.+\frac{1}{2} \int_{t}^{T} \sigma_{1 s}^{2} d s+k \int_{t}^{T} v_{s} d s\right\} .
\end{aligned}
$$

We define

$$
\begin{aligned}
\frac{d Q^{S}}{d P}= & \frac{e^{-\int_{t}^{T} r_{s} d s} S_{T}}{E\left[e^{-\int_{t}^{T} r_{s} d s} S_{T} \mid \mathscr{F}_{t}\right]} \\
= & \exp \left\{\int_{t}^{T} \sigma_{1 v} d W_{1 v}-\frac{1}{2} \int_{t}^{T} \sigma_{2 v}^{2} d v\right. \\
& \left.\quad+\int_{t}^{T} Y_{s-} d N(s)-k \int_{t}^{T} v_{s} d s\right\},
\end{aligned}
$$

and by Girsanov's theorem, under $Q^{S}$,

$$
\begin{gathered}
d W_{1 t}^{Q^{S}}=d W_{1 t}-\sigma_{1 t} d t \\
d W_{2 t}^{Q^{S}}=d W_{2 t}-\rho_{12} \sigma_{1 t} d t,
\end{gathered}
$$

and, under $Q^{S}, N_{t}$ is Poisson process with intensity $(k+1) v_{t}$, and the density function of $Y_{1}$ is $e^{y} f(y) /(k+1)$, where $f(\cdot)$ is the density function of $Y_{1}$ under $P$. Since, under $Q^{S}$,

$$
\begin{gathered}
S_{T}=S_{t} \exp \left\{\mathscr{A}(t, T)+\Delta+\int_{t}^{T} M(b, v, T) \sigma_{2 v} d W_{2 v}^{\mathrm{Q}^{S}}\right. \\
\left.+\int_{t}^{T} \sigma_{1 v} d W_{1 v}^{\mathrm{Q}^{S}}+\int_{t}^{T} Y_{s-} d N_{s}\right\},
\end{gathered}
$$

then

$$
\begin{gathered}
P^{Q^{S}}\left(S_{T} \geq K \mid \mathscr{F}_{t}\right) \\
=P^{Q^{S}}\left(\int_{t}^{T} \sigma_{1 s} d W_{1 s}^{Q^{S}}+\int_{t}^{T} \sigma_{2 s} M(b, s, T) d W_{2 s}^{Q^{S}}\right. \\
\left.\geq \ln \left(\frac{K}{S_{t}}\right)-\mathscr{A}(t, T)-\Delta-\int_{t}^{T} Y_{s-} d N_{s}\right) \\
=P^{Q^{T}}\left(\frac{\int_{t}^{T} \sigma_{1 s} d W_{1 s}^{Q^{S}}+\int_{t}^{T} \sigma_{2 s} M(b, s, T) d W_{2 s}^{Q^{S}}}{\sqrt{\Delta}}\right. \\
\left.\quad \leq \frac{\ln \left(S_{t} / K\right)+\mathscr{A}(t, T)+\Delta+\int_{t}^{T} Y_{s-} d N_{s}}{\sqrt{\Delta}}\right) \\
=\sum_{n=0}^{\infty} \frac{\left(\int_{t}^{T} v_{s} d s\right)^{n}}{n !} e^{-\int_{t}^{T} v_{s} d s} E^{S}\left(N\left(d_{4}\right)\right),
\end{gathered}
$$

where $E^{S}(\cdot)$ is the expectation of $Y_{j}$ under $Q^{S}$,

$$
\begin{aligned}
d_{4} & =\frac{\ln \left(S_{t} / K\right)+\mathscr{A}(t, T)+\Delta+\sum_{j=1}^{n} Y_{j}}{\sqrt{\Delta}} \\
& =d_{3}+\sqrt{\Delta} .
\end{aligned}
$$

From (42) and (33), we can conclude the following lemma.

Lemma 2. Consider the following:

$$
\begin{aligned}
& E\left[e^{-\int_{t}^{T} r_{s} d s}\left(S_{T}-K\right)^{+} \mid \mathscr{F}_{t}\right] \\
& =\sum_{n=0}^{\infty} \frac{\left(\int_{t}^{T} v_{s} d s\right)^{n}}{n !} \\
& \quad \times e^{-\int_{t}^{T} v_{s} d s}\left[A(t, T) E^{S}\left(N\left(d_{4}\right)\right)-K P(t, T) E\left(N\left(d_{3}\right)\right)\right] .
\end{aligned}
$$

Combined with the previous lemmas, the price of European call option at timet is given by the following theorem. 
TABLE 1: The parameter values.

\begin{tabular}{lcc}
\hline Parameter name & Value in state $e_{1}$ & Value in state $e_{2}$ \\
\hline Volatility of $S$ & $\sigma_{11}=0.2$ & $\sigma_{12}=0.4$ \\
Jump intensity & $\nu_{1}=15$ & $\nu_{2}=30$ \\
Speed of reversion of $r$ & $b_{1}=2$ & $b_{2}=1$ \\
Long-term average of $r$ & $a_{1} / b_{1}=0.04$ & $a_{2} / b_{2}=0.02$ \\
Volatility of $r$ & $\sigma_{21}=0.15$ & $\sigma_{22}=0.3$ \\
Speed of reversion of $\lambda$ & $\beta_{1}=1.5$ & $\beta_{2}=2$ \\
Long-term average of $\lambda$ & $\alpha_{1} / \beta_{1}=0.01$ & $\alpha_{2} \beta_{2}=0.02$ \\
Volatility of $\lambda$ & $\sigma_{31}=0.25$ & $\sigma_{32}=0.45$ \\
Initial stock price & $S_{0}=100$ & \\
Initial interest rate & $r_{0}=0.04$ & \\
Initial default intensity & $\lambda_{0}=0.5$ & \\
Mean jump size & $\mu_{J}=0$ & \\
Standard deviation of jump size & $\sigma_{J}=0.1$ & \\
\hline
\end{tabular}

Theorem 3. The price of European call option with credit risk at time $t$ is

$$
\begin{aligned}
& C(t, T) \\
& \qquad \sum_{n=0}^{\infty} \frac{\left(\int_{t}^{T} v_{s} d s\right)^{n}}{n !} \\
& \times e^{-\int_{t}^{T} v_{s} d s}\left[w \left(A(t, T) E^{S}\left(N\left(d_{4}\right)\right)\right.\right. \\
& - \\
& \left.+K P(t, T) E\left(N\left(d_{3}\right)\right)\right) \\
& +(1-w) 1_{(\tau>t)} Z(t, T) \\
& \times\left(\mathscr{L}(t, T) E^{R}\left(N\left(d_{2}\right)\right)\right. \\
& \\
& \left.\left.-K E\left(N\left(d_{1}\right)\right)\right)\right] .
\end{aligned}
$$

\section{Numerical Analysis}

In this section, we will employ Monte Carlo simulation and perform a numerical analysis for European-style call option with credit risk under the Markov-modulated jump-diffusion model. We consider that the Markov chain $\xi$ has two states, which means that macroeconomic shifts between the two states: $e_{1}$ ("boom" or "good") and $e_{2}$ ("recession" or "bad"). We assume that the current economy is in boom and that the transition probability matrix of the two-state Markov chain $\xi$ is given by

$$
\left(\begin{array}{ll}
p_{11} & p_{12} \\
p_{21} & p_{22}
\end{array}\right)=\left(\begin{array}{ll}
0.7 & 0.3 \\
0.2 & 0.8
\end{array}\right)
$$

Assume that $Y_{t}$ is normally distributed with mean $\mu_{J}$ and standard deviation $\sigma_{J}$. We will adopt the specimen values for the model parameters as Table 1 . We consider a range of spot-to-strike ratios $S_{0} / K$ from 0.8 to 1.2 and assume that one year has 252 trading days. We perform 10000 simulations for computing the option price.

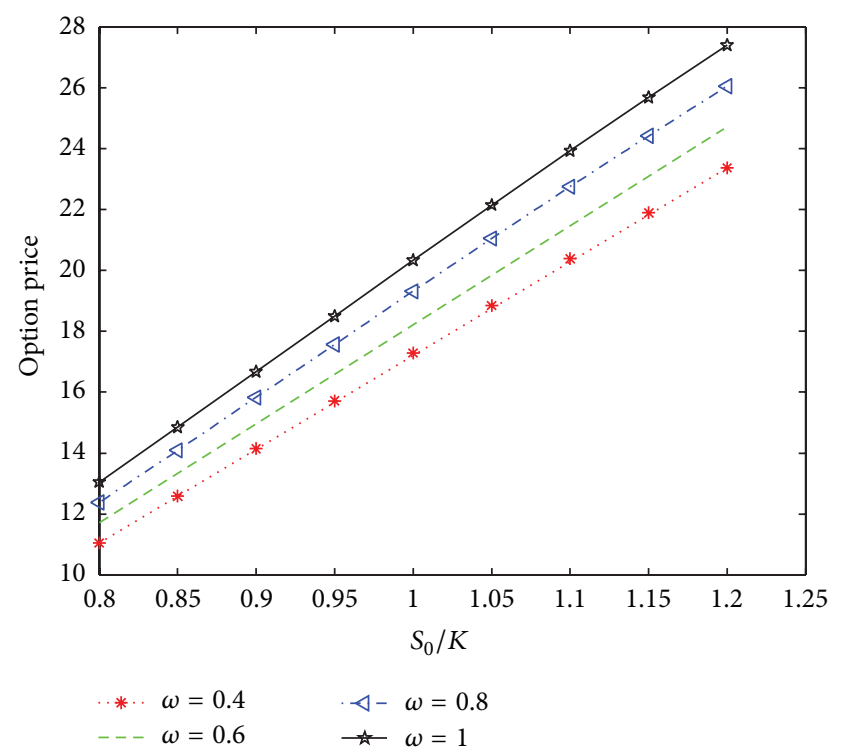

FIGURE 1: The option price with different recovery rate for $T=1$, $\rho_{12}=0.7, \rho_{13}=0.5$, and $\rho_{23}=0.6$.

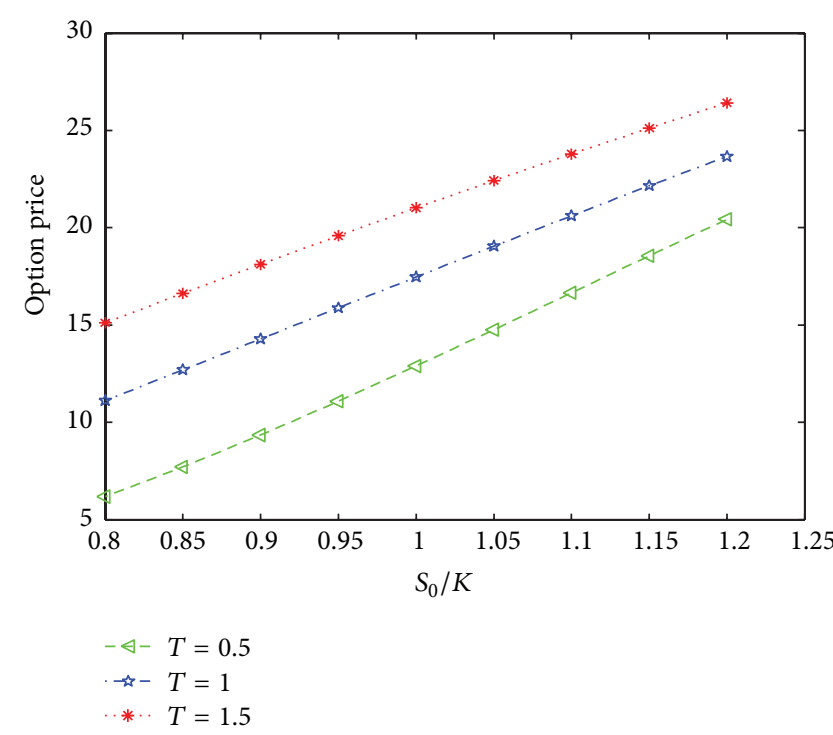

FIgURE 2: The option price with different maturity $T$ for $\omega=0.4$, $\rho_{12}=0.7, \rho_{13}=0.5$, and $\rho_{23}=0.6$.

For each $\omega=0.4,0.6,0.8,1$, we consider the impact of the spot-to-strike ratio on the option price. From Figure 1, we observe that the option price increases as the spot-to-strike ratio increases. We can also see that the greater the $\omega$, the greater the option price. When $\omega=1$, it follows that there is not default risk. It is a result of the fact that the payoff at the maturity will increase as the recovery rate increases. Figure 2 depicts the plot of the option price against the spotto-strike ratio for each maturity $T=0.5,1,1.5$. From these plots, we find that the longer the maturities, the greater the option price.

In the following, we compare the option price with different correlation coefficients for fixed maturity $T=1$ 


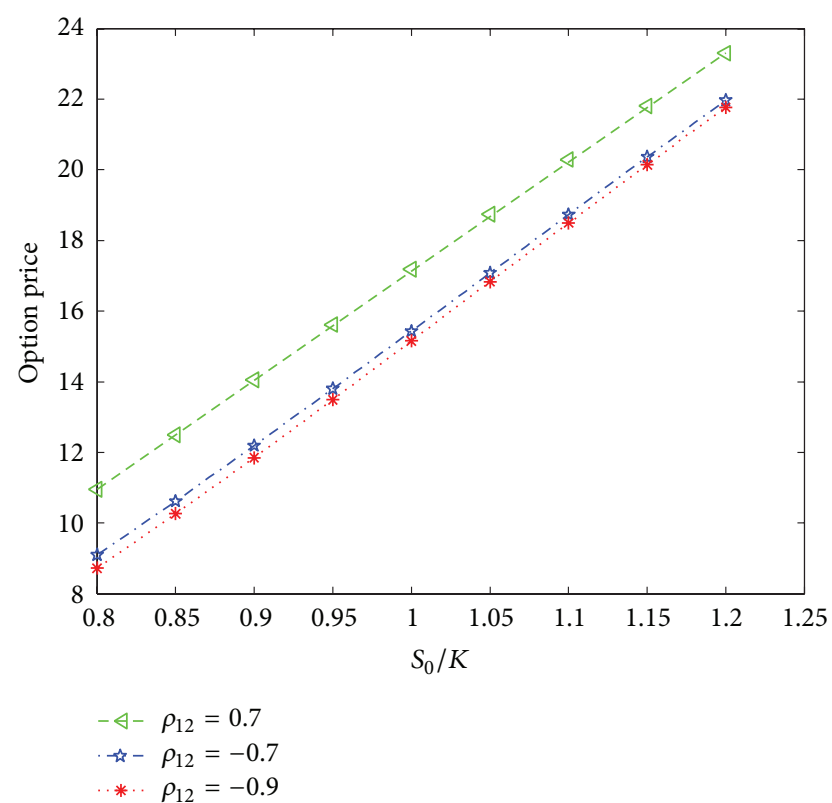

FIGURE 3: The option price with different correlation coefficients $\rho_{12}$ for $T=1, \omega=0.4, \rho_{13}=0.5$, and $\rho_{23}=0.6$.

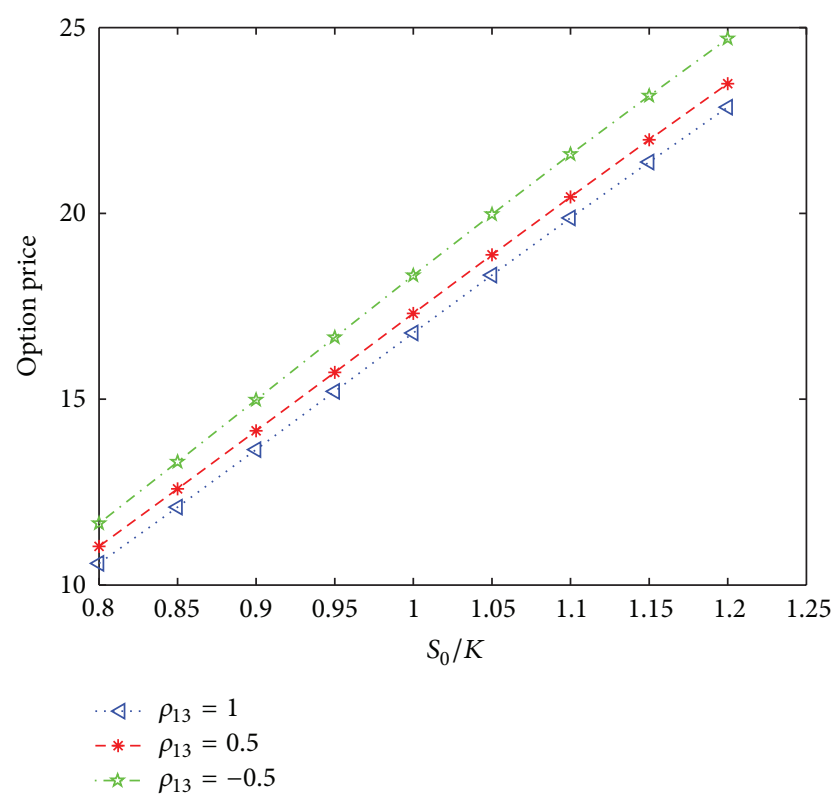

FIgURE 4: The option price with different correlation coefficients $\rho_{13}$ for $T=1, \omega=0.4, \rho_{12}=0.7$, and $\rho_{23}=0.6$.

and recovery rate $\omega=0.4$. Figure 3 illustrates that option price increases as correlation coefficient $\rho_{12}$ increases. From Figure 4 , we can also see that option price decreases as correlation coefficient $\rho_{13}$ increases.

In Figure 5, we present how the option prices vary with the changes of the annual jump intensity $v$. Figure 5 displays a large change in the option price due to the variation of the jump intensity. The option price increases as jump intensity increases.

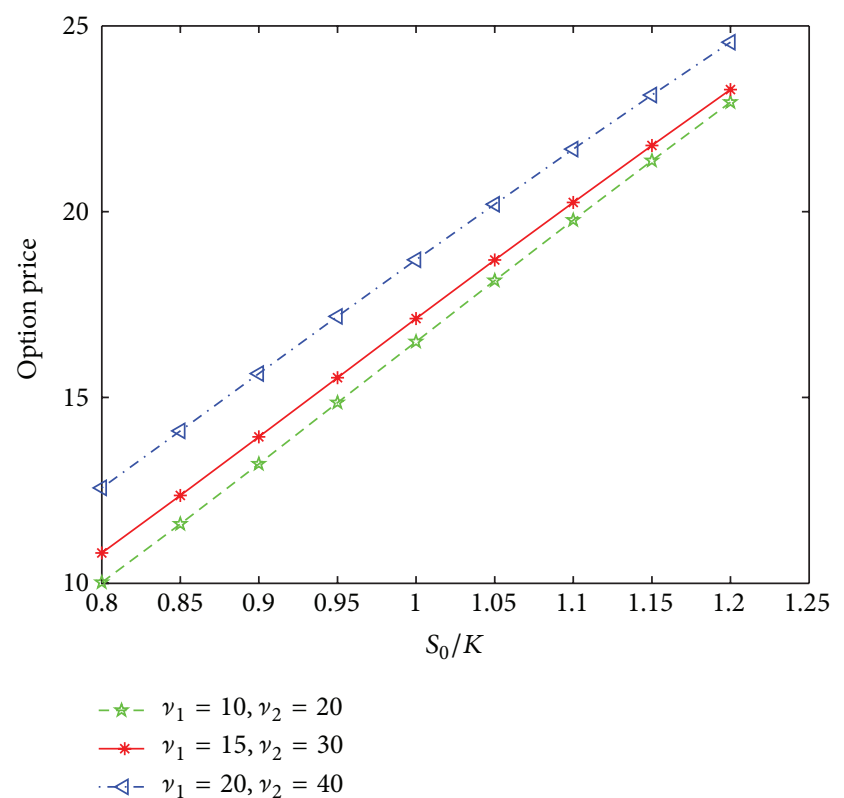

FIGURE 5: The option price with different $v$ for $\omega=0.4, \rho_{12}=0.7$, $\rho_{13}=0.5$, and $\rho_{23}=0.6$.

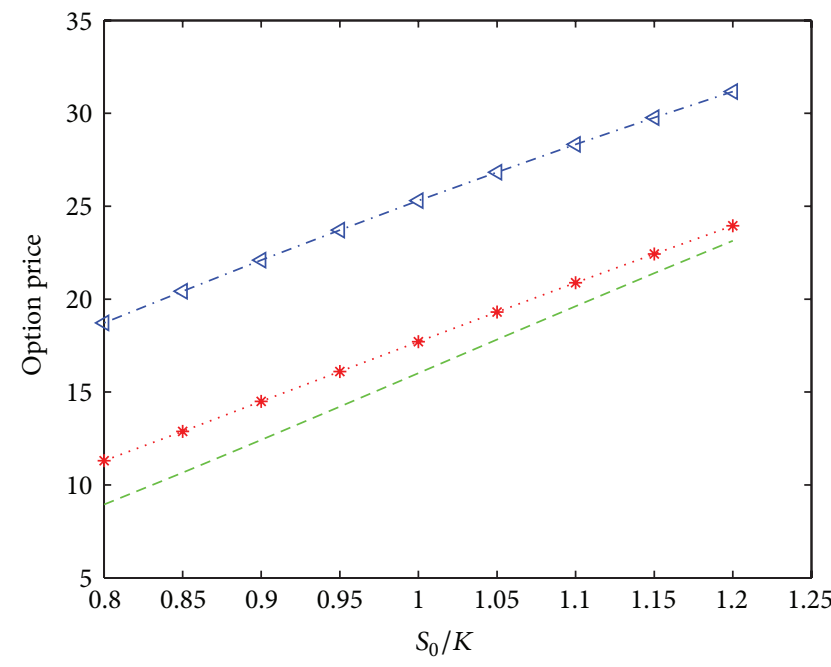

..*. Markov-modulated model
--- Non-Markov-modulated model for a good economy
४- Non-Markov-modulated model for a bad economy

FIGURE 6: The option price in Markov-modulated model and nonMarkov-modulated models.

Finally, we investigate the difference of the option price in Markov-modulated model and non-Markov-modulated models. From Figure 6, we can observe that the call option price in good economy is lower than that in bad economy. The reason is that when economy is bad, the volatility of the risky asset is great so that the option price is higher. In our model, we assume that the current economy is good, so Markovmodulated model is close to the model for a good economy, while the two plots and the model for a bad economy are relatively far apart. In Markov-modulated model, we take into 
consideration the changes of the state of the economy, so Markov-modulated model is more in accord with the reality for the pricing of the defaultable options.

\section{Conclusion}

The pricing of European option with credit risk in a reduced form model was studied, while the stock price was driven by Markov-modulated jump-diffusion models. The interest rate and the default intensity followed the Vasicek models, and the parameters were also controlled by the same Markov chain. Compared with most of the credit risk models, the main advantage is that we incorporated Markov-modulated rates into the models. We applied Girsanov's theorem to obtain the equivalent martingale measure and derived the closed form formula for the valuation of the European option. Finally, from the numerical illustrations, we obtain the effects of the recovery rate, the maturity, the correlation coefficients, and the jump intensity of stock on the option price.

\section{Acknowledgments}

The authors would like to thank the referee for many helpful and valuable comments and suggestions. Jinzhi Li would like to acknowledge the National Commission of Ethnic Affair (no. 12ZYZ003) and the Ministry of Education of Humanities and Social Sciences Project (no. 11YJC790102).

\section{References}

[1] R. Merton, "On the pricing of corporate debt: the risk structure of interest rates," The Journal of Finance, vol. 29, no. 2, pp. 449470, 1974.

[2] M. Ammann, Credit Risk Valuation: Methods, Models, and Applications, Springer, New York, NY, USA, 2nd edition, 2001.

[3] P. Klein and M. Inglis, "Pricing vulnerable European options when the option's payoff can increase the risk of financial distress," Journal of Banking and Finance, vol. 25, no. 5, pp. 9931012, 2001.

[4] P. Artzner and D. Freddy, "Default risk insurance and incomplete markets," Mathematical Finance, vol. 5, no. 3, pp. 187-195, 1995.

[5] D. Duffie and K. J. Singleton, "Modeling term structures of defaultable bonds," Review of Financial Studies, vol. 12, no. 4, pp. 687-720, 1999.

[6] D. Duffie and N. Gârleanu, "Risk and valuation of collateralized debt obligations," Financial Analysts Journal, vol. 57, no. 1, pp. 41-59, 2001.

[7] K. S. Leung and Y. K. Kwok, "Counterparty risk for credit default swaps: Markov chain interacting intensities model with stochastic intensity," Asia-Pacific Financial Markets, vol. 16, no. 3, pp. 169-181, 2009.

[8] X. Su and W. Wang, "Pricing options with credit risk in a reduced form model," Journal of the Korean Statistical Society, vol. 41, no. 4, pp. 437-444, 2012.

[9] R. J. Elliott, W. C. Hunter, and B. M. Jamieson, "Financial signal processing: a self calibrating model," International Journal of Theoretical and Applied Finance, vol. 4, no. 4, pp. 567-584, 2001.
[10] R. J. Elliott, L. Chan, and T. K. Siu, "Option pricing and Esscher transform under regime switching," Annals of Finance, vol. 1, no. 4, pp. 423-432, 2005.

[11] R. J. Elliott and C. J. Osakwe, "Option pricing for pure jump processes with Markov switching compensators," Finance and Stochastics, vol. 10, no. 2, pp. 250-275, 2006.

[12] R. J. Elliott, T. K. Siu, L. Chan, and J. W. Lau, "Pricing options under a generalized Markov-modulated jump-diffusion model," Stochastic Analysis and Applications, vol. 25, no. 4, pp. 821-843, 2007.

[13] T. K. Siu, H. L. Yang, and J. W. Lau, "Pricing currency options under two-factor Markov-modulated stochastic volatility models," Insurance, vol. 43, no. 3, pp. 295-302, 2008.

[14] L. J. Bo, Y. J. Wang, and X. W. Yang, "Markov-modulated jumpdiffusions for currency option pricing," Insurance, vol. 46, no. 3 , pp. 461-469, 2010.

[15] D. Lando, “On cox processes and credit risky securities," Review of Derivatives Research, vol. 2, no. 2-3, pp. 99-120, 1998. 


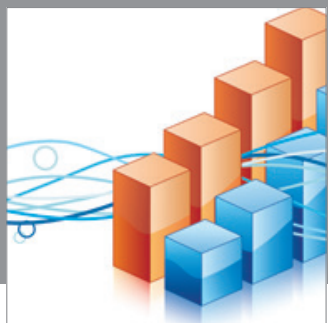

Advances in

Operations Research

mansans

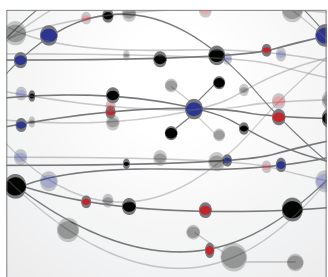

The Scientific World Journal
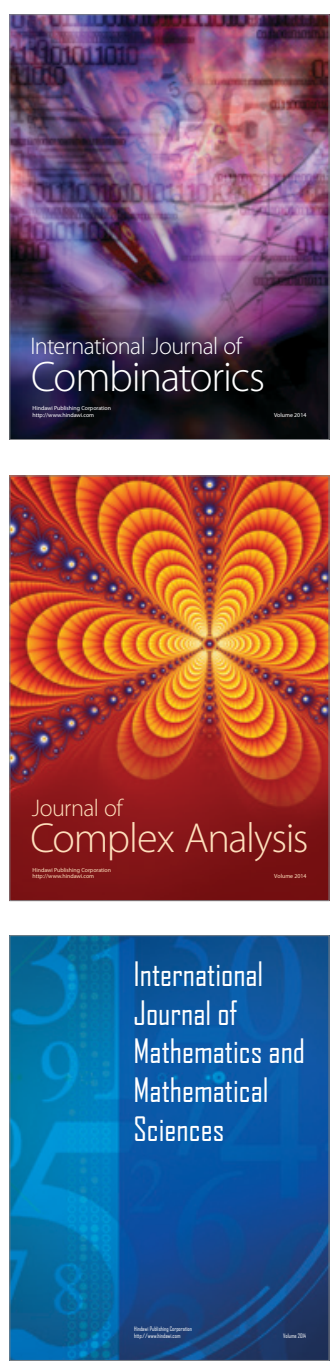
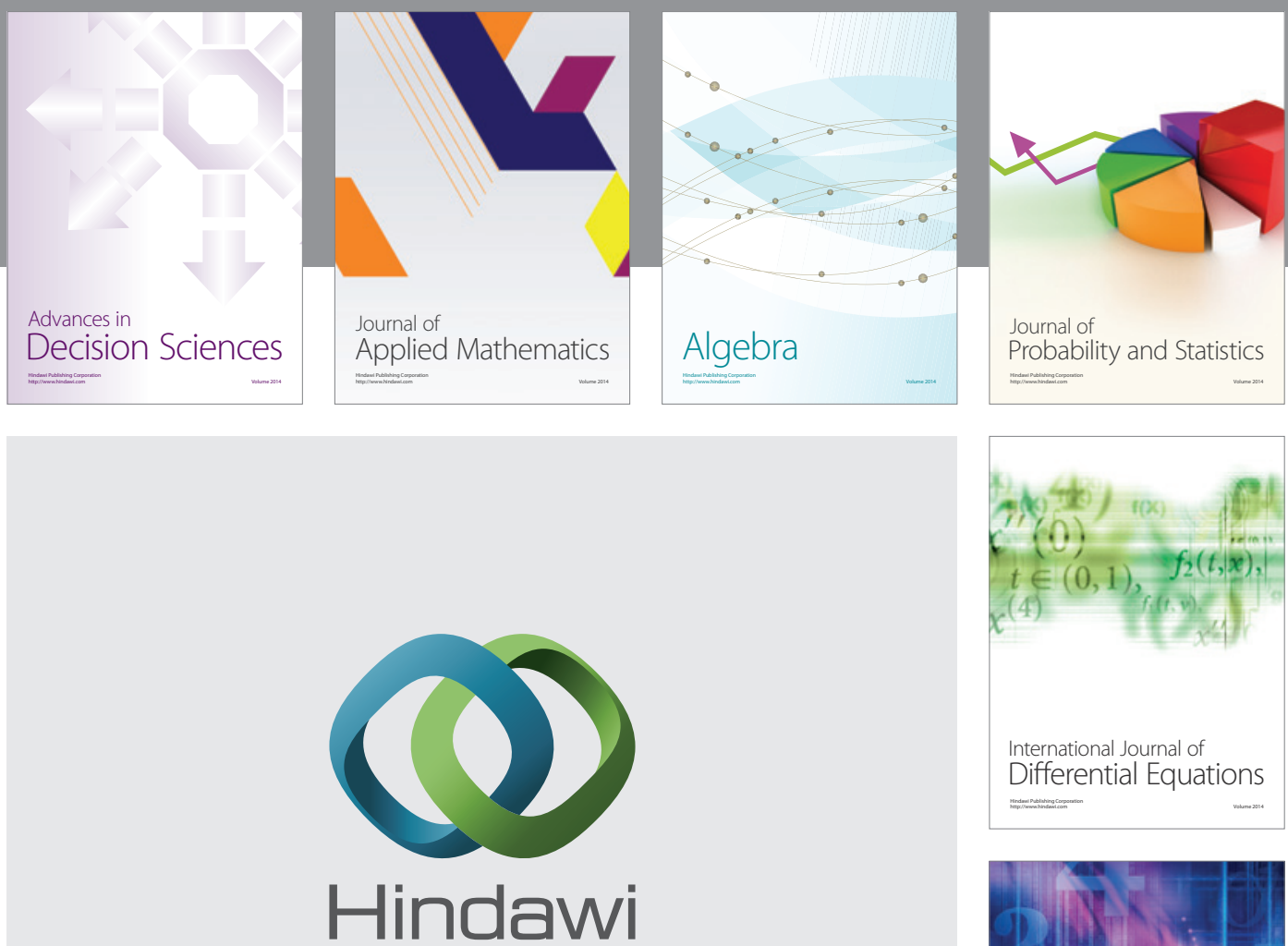

Submit your manuscripts at http://www.hindawi.com
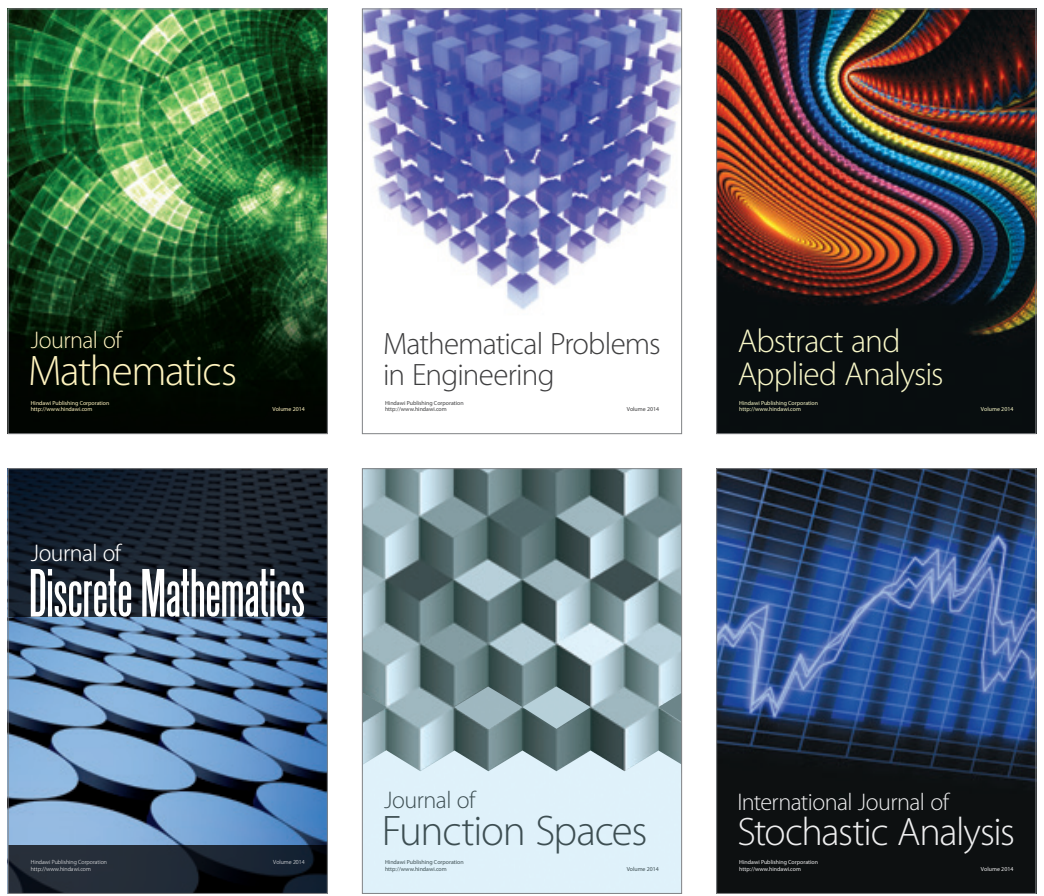

Journal of

Function Spaces

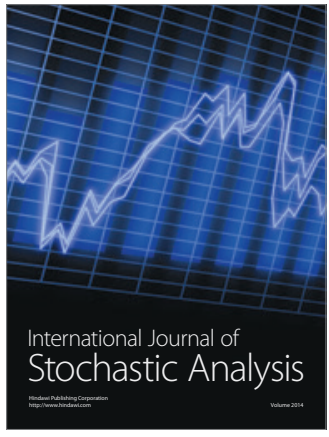

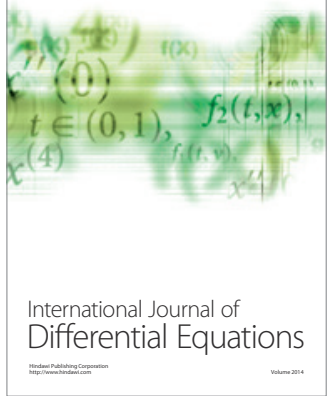
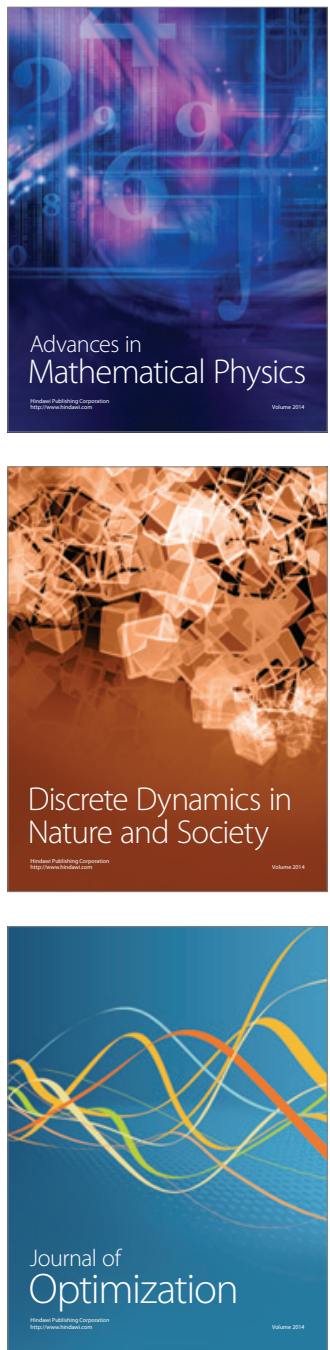